\title{
Two dichotomy theorems on colourability of non-analytic graphs
}

\author{
by
}

\section{Vladimir Ka novei (Moscow)}

\begin{abstract}
We prove:
THEOREM 1. Let $\kappa$ be an uncountable cardinal. Every $\kappa$-Suslin graph $\mathrm{G}$ on reals satisfies one of the following two requirements: (I) G admits a $(\kappa+1)$-Borel colouring by ordinals below $\kappa$; (II) there exists a continuous homomorphism (in some cases an embedding) of a certain locally countable Borel graph $\mathrm{G}_{0}$ into $\mathrm{G}$.

TheOREM 2. In the Solovay model, every OD graph G on reals satisfies one of the following two requirements: (I) G admits an $\mathrm{OD}$ colouring by countable ordinals; (II) as above.
\end{abstract}

Introduction. A new direction in the theory of graphs was discovered by Kechris, Solecki, and Todorčević [9]. They demonstrated that graphs on reals change their behaviour in the case when a definable colouring is required. For instance, there exists a Borel locally countable and acyclic (hence colourable by only two colours) graph $\mathrm{G}_{0}$ on reals (or rather a family of graphs generated by a common method) which does not admit a countable Borel colouring.

To get such a graph, let us fix once and for all a binary tuple $\mathfrak{s}_{m} \in 2^{m}$ for each $m \in \omega$, so that for any $s \in 2^{<\omega}$ we have $s \subseteq \mathfrak{s}_{m}$ for some $m$ (the density). We set, for $x, y \in 2^{\omega}, x \mathrm{G}_{0} y$ iff there exists $m$ satisfying

$$
x\left\lceil m=y\left\lceil m=\mathfrak{s}_{m}, \quad x(m)=1-y(m), \quad \text { and } \quad x(k)=y(k) \text { for all } k>m .\right.\right.
$$

Thus $\mathrm{G}_{0}$ is a Borel (more exactly, $\mathbf{F}_{\sigma}$ ) locally countable acyclic $\left({ }^{1}\right.$ ) graph on the Cantor space $\mathcal{D}=2^{\omega}$. An argument of Kechris et al. [9] shows that the density property implies countable Borel uncolourability of $\mathrm{G}_{0}$.

1991 Mathematics Subject Classification: 03E15, 03E35, 04A15.

Partially supported by DFG, NWO, and the Carlsberg foundation.

$\left({ }^{1}\right)$ A (symmetric irreflexive) graph $\mathrm{G}$ is acyclic iff there does not exist a path of the form $x_{0} \mathrm{G} x_{1} \mathrm{G} x_{2} \mathrm{G} \ldots \mathrm{G} x_{n}$ where $n \geq 3, x_{0}=x_{n}$, and $x_{i} \neq x_{j}$ except for $i=0, j=n$. A graph $\mathrm{G}$ is locally countable iff every vertex has at most countably many G-neighbours. 
Moreover, it is proved in [9] that $\mathrm{G}_{0}$ is in a certain sense minimal among all $\boldsymbol{\Sigma}_{1}^{1}$ graphs which are not countably Borel colourable $\left({ }^{2}\right)$.

To formulate this result consistently, let us recall some notation. We refer to [9] for a more substantial review and to [8] as a broad reference.

Let $\mathrm{G}$ be a graph on a set $X$. A set $Y \subseteq X$ is $\mathrm{G}$-discrete iff $\mathrm{G}$ does not intersect $Y \times Y$. G is countably Borel colourable iff $X$ is a union of countably many Borel G-discrete sets.

In general a colouring of a graph $\mathrm{G}$ on $X$ is a function $\phi$ defined on $X$ so that $x \mathrm{G} y$ implies $\phi(x) \neq \phi(y)$ (adjacent vertices are coloured differently). If, for instance, $X=\bigcup_{\alpha<\kappa} X_{\alpha}$ is a union of G-discrete sets $X_{\alpha}$ then the map $\phi$ which sends every $x \in X$ to the least ordinal $\alpha=\phi(x)<\kappa$ such that $x \in X_{\alpha}$ is a colouring.

Let $\mathrm{G}$ and $\mathrm{G}^{\prime}$ be graphs on sets $X$ and $X^{\prime}$ resp. A homomorphism of $\mathrm{G}^{\prime}$ into $\mathrm{G}$ is a function $H: X^{\prime} \rightarrow X$ such that $x \mathrm{G}^{\prime} y \Rightarrow H(x) \mathrm{G} H(y)$ for all $x, y \in X$. An embedding is a 1-1 homomorphism (but the opposite implication $H(x) \mathrm{G} H(y) \Rightarrow x \mathrm{G}^{\prime} y$ is not required).

Let $X$ and $X^{\prime}$ be topological spaces. One writes $\mathrm{G}^{\prime} \leq_{\mathrm{c}} \mathrm{G}$ if there exists a continuous homomorphism of $G^{\prime}$ into $G$. One writes $G^{\prime} \sqsubseteq_{c} G$ if there exists a continuous embedding of $G^{\prime}$ into $G$. (c stands for "continuous".)

Even $G^{\prime} \leq_{c} G$ suffices for $G$ to inherit uncolourability properties from $G^{\prime}$. In particular, it follows from the above that any graph $G$ satisfying $G_{0} \leq_{c} G$ is countably Borel uncolourable. The next theorem of Kechris, Solecki, and Todorčević [9] shows that this is also a necessary condition.

TheOREM 1 (Theorem 6.3 in [9]). Every $\boldsymbol{\Sigma}_{1}^{1}$ graph $\mathrm{G}$ on reals satisfies one and only one of the two statements, (I) and (II):

(I) G is countably Borel colourable. Moreover, if G is a $\Sigma_{1}^{1}\left(z_{0}\right)$ graph, $z_{0}$ being a real, then each real belongs to a $\mathrm{G}$-discrete $\Delta_{1}^{1}\left(z_{0}\right)$ set.

(II) $\mathrm{G}_{0} \leq_{\mathrm{c}} \mathrm{G}$. Moreover, $\mathrm{G}_{0} \sqsubseteq_{\mathrm{c}} \mathrm{G}$ in each of the two cases: (a) $\mathrm{G}$ is acyclic; (b) $\mathrm{G}$ is locally countable.

By reals we understand, as usual, points of the Baire space $\mathcal{N}=\omega^{\omega}$.

The theorem is not true for $\boldsymbol{\Pi}_{1}^{1}$ graphs $\left({ }^{3}\right)$. On the other hand, there is a reasonable generalization on Suslin graphs. Recall that a set $G \subseteq \mathcal{N}^{2}$ is

$\left({ }^{2}\right)$ Essential properties of $\mathrm{G}_{0}$ do not depend on a particular choice of the tuples $\mathfrak{s}_{m}$. However, it seems unknown whether different graphs of the form $\mathrm{G}_{0}$ are isomorphic.

$\left({ }^{3}\right)$ Indeed, consider the $\Pi_{1}^{1}$ graph $\mathrm{G}$ of all pairs of reals $\langle x, y\rangle$ such that $x$ and $y$ code ordinals and the ordinal coded by $x$ differs from the ordinal coded by $y$. Clearly $\mathrm{G}$ includes a full $\aleph_{1}$-subgraph, therefore is countably uncolourable. If now $H$ is a Borel homomorphism of $\mathrm{G}_{0}$ in $\mathrm{G}$ then by a classical restriction theorem $H$ involves only countably many ordinals, so $G_{0}$ would be countably Borel colourable, which is a contradiction. Kechris et al. give in [9] more substantial examples. 
$\lambda$-Suslin iff there is a tree $R \subseteq(\omega \times \omega \times \lambda)^{<\omega}$ such that

$$
\mathrm{G}=\mathfrak{p}[R]=\left\{\langle x, y\rangle: \exists f \in \lambda^{\omega}[R](x, y, f)\right\},
$$

where $[R]=\left\{\langle x, y, f\rangle \in \mathcal{N} \times \mathcal{N} \times \lambda^{\omega}: \forall m R(x\lceil m, y\lceil m, f\lceil m)\}\right.$.

Theorem 2. Let $\kappa \geq \aleph_{1}$ be a cardinal. Every $\kappa$-Suslin graph $\mathrm{G}$ on reals satisfies one $\left({ }^{4}\right)$ of the following two statements:

(I) $\mathrm{G}$ admits a $(\kappa+1)$-Borel colouring by ordinals below $\kappa$. Moreover, if $\kappa \leq \lambda<\kappa^{+}, R \subseteq(\omega \times \omega \times \lambda)^{<\omega}$, and $\mathrm{G}=\mathfrak{p}[R]$, then there is a sequence $\left\langle C_{\alpha}: \alpha<\lambda\right\rangle \in \mathrm{L}[R]$ of $(\lambda+1)$-Borel codes such that each real belongs to a $\mathrm{G}$-discrete $(\lambda+1)$-Borel set coded by some $C_{\alpha}$.

(II) $\mathrm{G}_{0} \leq_{\mathrm{c}} \mathrm{G}$. Moreover, $\mathrm{G}_{0} \sqsubseteq_{\mathrm{c}} \mathrm{G}$ in each of the two cases: (a) $\mathrm{G}$ is acyclic; (b) $\mathrm{G}$ is locally thin $\left({ }^{5}\right)$.

(As usual, a $(\lambda+1)$-Borel set is a set which belongs to the smallest family containing all open sets and closed under $\lambda$-size unions and intersectionsthis is the same as $\kappa^{+}$-Borel provided $\kappa \leq \lambda<\kappa^{+}$.)

Thus (I) of Theorem 2 says that the reals are covered by a reasonably effective union of $\kappa$-many G-discrete $\kappa^{+}$-Borel sets.

Problem 1. Can (I) be strengthened in Theorem 2 to a colouring by $\kappa$-many $\kappa$-Borel G-discrete sets? This is open even in the most interesting case $\kappa=\omega_{1}$, which includes the case of $\Sigma_{2}^{1}$ graphs.

Problem 2. Find reasonable counterexamples among $\boldsymbol{\Pi}_{2}^{1}$ graphs.

Problem 3. Characterize countably colourable graphs in Theorem 2.

It is a common practice that theorems on Borel, analytic etc. sets generalize in the Solovay model $\left({ }^{6}\right)$ on ROD (real-ordinal definable) sets. The following theorem is such a generalization of Theorems 1 and 2 .

TheOREM $3\left({ }^{7}\right)$. In the Solovay model, every ROD graph G on reals satisfies one and only one of the two statements, (I) and (II):

$\left({ }^{4}\right)$ In this case (I) and (II) are generally speaking compatible. For instance assuming the axiom of constructibility every graph admits a $\Delta_{2}^{1}$ (in the codes) colouring by countable ordinals via a $\Delta_{2}^{1}$ bijection between $\omega_{1}$ and the reals.

$\left({ }^{5}\right)$ A thin set is a set which does not contain a perfect subset. A graph $\mathrm{G}$ is locally thin if for any vertex $x$ the set of all G-neighbours of $x$ is thin. This includes for instance locally countable graphs.

$\left({ }^{6}\right)$ The Solovay model is a model of ZFC, defined by Solovay [10], where all projective sets are Lebesgue measurable.

$\left({ }^{7}\right)$ It is mentioned in [9] with a reference to Woodin that a similar dichotomy theorem is a consequence of a determinacy hypothesis. 
(I) $\mathrm{G}$ admits a colouring by countable ordinals, ROD in $\mathrm{HC}\left({ }^{8}\right)$. Moreover, if $\mathrm{G}$ is an $\mathrm{OD}\left(z_{0}\right)$ graph, $z_{0}$ being a real, then each real belongs to a $\mathrm{G}$-discrete Borel set with a code in $\mathrm{L}\left[z_{0}\right]$.

(II) $\mathrm{G}_{0} \leq_{\mathrm{c}} \mathrm{G}$. Moreover, $\mathrm{G}_{0} \sqsubseteq_{\mathrm{c}} \mathrm{G}$ in each of the two cases: (a) $\mathrm{G}$ is acyclic; (b) $\mathrm{G}$ is locally thin (then in fact $\mathrm{G}$ is locally countable).

(A code in (I) of this theorem is understood to code the construction of a Borel set by operations which are countable, but not necessarily countable in $\mathrm{L}\left[z_{0}\right]$.)

Problem 4. Prove Theorems 2 and 3 with (II) in the form $G_{0} \sqsubseteq_{c} G$ for all relevant graphs $\mathrm{G}$. (This is also open for Theorem 1.)

Our preprint [7] contains preliminary versions of Theorems 2 and 3. (The former much weaker than Theorem 2 here, in particular we consider in [7] only $\Sigma_{2}^{1}$ graphs and require that the universe is a generic extension of L, the constructible universe.) The proofs in [7] involve a technique quite close to arguments in [9] but with the topology generated by OD sets (similarly to [6] where the OD topology is applied for a problem of classification of ROD equivalence relations in the Solovay model) rather than the GandyHarrington topology as in [9]. We choose here a different approach.

The major part of the paper is devoted to Theorem 2 .

The proof makes use of a method outlined in a note by Hjorth [5], who attributes the technique to Harrington and Shelah [3].

Starting the proof, we fix an ordinal $\lambda, \kappa \leq \lambda<\kappa^{+}$, and a tree $R \subseteq$ $(\omega \times \omega \times \lambda)^{<\omega}$ satisfying $\mathbf{G}=\mathfrak{p}[R]$. To simplify the notation we suppose that actually $R \in \mathrm{L}$ (otherwise $\mathrm{L}$ uniformly changes to $\mathrm{L}[R]$ in the reasoning).

The idea is similar to several other recent dichotomy results in descriptive set theory (see for example Harrington et al. [2]): either the union of some suitably regular sets covers the reals, easily leading to a (I)-like case, or otherwise we have enough singularity to work towards a (II)-like case, which is usually more difficult. So the initial point is to choose the covering sets.

We take G-discrete $(\lambda+1)$-Borel sets coded in $\mathrm{L}$ to be the covering sets, but, to avoid triviality, consider the covering property in a suitably comprehensive generic extension $\mathrm{V}^{+}$of the basic set universe $\mathrm{V}$.

Section 2 introduces the dichotomy: either every real in $\mathrm{V}^{+}$belongs to a G-discrete set having a $(\lambda+1)$-Borel code in $\mathrm{L}$, or this is not the case.

In the either case a kind of compactness argument reduces the number of covering sets to $\lambda$ leading to a partition of the reals into $\lambda$-many $\mathrm{G}$-discrete $(\lambda+1)$-Borel sets in the original universe; see Section 3. The partition we define is effective: there is a constructible $\lambda$-sequence of (also constructible) $(\lambda+1)$-Borel codes for sets in the partition.

$\left({ }^{8}\right) \mathrm{HC}$ is the set of all hereditarily countable sets. 
Sections 4 and 5 handle the or case. We show that then $G_{0} \leq_{c} G$, even $\mathrm{G}_{0} \sqsubseteq_{\mathrm{c}} \mathrm{G}$ provided $\mathrm{G}$ is acyclic or locally thin. This argument involves a splitting construction of Kechris et al. [9], but technically realized in a different way: instead of making use of Choquet games we all the time keep watching to guarantee that each level of the splitting system contains, in $\mathrm{V}^{+}$, reals not covered by the above-mentioned discrete sets.

Section 6 presents a proof of Theorem 3 which involves an appropriate modification of the basic technique.

Theorem 2 has a special case, the case of $\boldsymbol{\Sigma}_{2}^{1}$ graphs, which admits a much simpler proof (of indeed a weaker result) based on Theorem 1 . We outline this argument, close to a proof of the theorem that a $\boldsymbol{\Sigma}_{1}^{1}$ equivalence relation has either $\leq \aleph_{1}$ or $\mathfrak{c}$ equivalence classes by Burgess, in Section 7 .

We finally demonstrate, in Section 8, that the basic technique is also applicable for $\boldsymbol{\Sigma}_{1}^{1}$ graphs (the subject of Theorem 1).

The exposition starts with a review and development of some facts related to coding of Borel and Suslin sets, in particular a version of the classical separation theorem, in Section 1.

1. Constructible coding of Borel and Suslin sets. The proof of the "lightface" $(R \in \mathrm{L})$ version of Theorem 2 will make use of sets having Borel and Suslin codes in L. (The general case changes this to L $[R]$.) This section introduces an appropriate formalism.

We shall consider spaces presented in the form $X=B^{\omega}$, where $B \in \mathrm{L}$, as e.g. the Baire space $\mathcal{N}=\omega^{\omega}$, or the product $\mathcal{N} \times \mathcal{N}$ identified with $(\omega \times \omega)^{\omega}$, or $\lambda^{\omega}$ where $\lambda \in \operatorname{Ord}$. If $\mathcal{X}=B^{\omega}$ is such a space and $u \in B^{<\omega}$ then we define $X_{u}=\{x \in \mathcal{X}: u \subset x\}$, a basic clopen set in $X$.

1.1. Borel and Suslin codes. Let $\lambda \in$ Ord. A $\lambda$-Borel code for a space $X=B^{\omega}$ is a pair of the form $C=\langle T, F\rangle$, where $T \subseteq \nu^{<\omega}$ for some $\nu<\lambda$ is a non-empty well-founded tree (in particular $s^{\wedge} \gamma \in T$ implies $s \in T$ for all $s \in \nu^{<\omega}$ and $\gamma<\nu$ ), and $F$ maps the collection $\operatorname{Max} T$ of all $\subseteq$-maximal elements of $T$ into $B^{<\omega}$.

If $C=\langle T, F\rangle$ is a $\lambda$-Borel code then a set $[C]_{t} \subseteq X$ is defined for each $t \in T$ by induction on the rank of $t$ in $T$, as follows:

- if $t \in \operatorname{Max} T$ then $[C]_{t}=X_{F(t)}=\{x \in X: F(t) \subset x\}$;

- if $t \in T \backslash \operatorname{Max} T$ then $[C]_{t}=\complement \bigcup_{u^{\wedge} \gamma \in T}[T]_{u^{\wedge} \gamma}$.

( $\complement$ denotes the complement.) Finally, we define $[C]=[C]_{\Lambda}$. ( $\Lambda$ is the empty sequence.) Sets of this form are called $\lambda$-Borel sets.

Classically Suslin sets are defined as projections of closed sets. In what follows we will make use of projections of $\mathbf{G}_{\delta}$ sets, which is essentially one and the same class of course, but the associated coding is much more flexible. We shall add weak to the relevant notation. 
By a weak $\lambda$-Suslin code for a space $X=B^{\omega}$ we understand an indexed family $\mathrm{d}=\left\langle D_{\xi}: \xi<\lambda^{\prime}\right\rangle$, where $\lambda^{\prime} \leq \lambda+\omega$, of sets $D_{\xi} \subseteq(B \times \lambda)^{<\omega}$. Let

$$
[\mathrm{d}]=\left\{\langle x, a\rangle \in \mathcal{X} \times \lambda^{\omega}: \forall \xi<\lambda^{\prime} \exists m\left(\langle x| m, a\lceil m\rangle \in D_{\xi}\right)\right\}
$$

and define the projection $\mathfrak{p}[\mathrm{d}]=\{x \in \mathcal{X}: \exists a[\mathrm{~d}](x, a)\}$. Sets of the form $\mathfrak{p}[\mathrm{d}]$, $\mathrm{d}$ being a weak $\lambda$-Suslin code, are called weak $\lambda$-Suslin sets.

We shall be especially interested in constructible codes. Sets of the form $[C]$ where $C \in \mathrm{L}$ is a $\lambda$-Borel code will be called $\lambda$-L-Borel sets. Sets of the form $\mathfrak{p}[\mathrm{d}]$ where $\mathrm{d} \in \mathrm{L}$ is a weak $\lambda$-Suslin code will be called weak $\lambda$-L-Suslin sets. ( $\mathrm{L}$ is the class of all constructible sets.)

Lemma 4. If $\lambda<\omega_{1}$ then any $(\lambda+1)$-L-Borel set is weak $\lambda$-L-Suslin.

Proof. Let $C=\langle T, F\rangle \in \mathrm{L}$ be a $(\lambda+1)$-Borel code for a space $\mathcal{X}=B^{\omega}$; $T \subseteq \lambda^{<\omega}$. Let $x \in \mathcal{X}$. Define a map $h_{x}: T \rightarrow\{0,1\}$ by $h_{x}(t)=1$ iff $x \in[C]_{t}$, for all $t \in T$. Then $x \in[C]$ iff $h_{x}(\Lambda)=1$. Consider the set

$P=\left\{\langle x, b, a\rangle \in \mathcal{X} \times T^{\omega} \times 2^{\omega}: b\right.$ is a bijection from $\omega$ onto $T \&$

$$
\left.a * b^{-1}=h_{x} \& a\left(b^{-1}(\Lambda)\right)=1\right\}
$$

where $\left(a * b^{-1}\right)(t)=a\left(b^{-1}(t)\right)$ for all $t \in T$. One easily sees that $[C]$ is the projection of $P$, so that $[C]=\{x \in X: \exists b \exists a P(x, b, a)\}$. On the other hand, the property of being equal to $h_{x}$ can be expressed by a $\Pi_{2}^{0}$-like statement, leading to a weak $\lambda$-Suslin code $\mathrm{d} \in \mathrm{L}$ satisfying $P=[\mathrm{d}]$.

1.2. Constituents and separation. This subsection shows that some classical constructions in the theory of Suslin sets have their counterparts in the "weak" setting.

Let $\lambda, \lambda^{\prime} \in$ Ord, $\lambda^{\prime} \leq \lambda+\omega$.

Consider a tree $T \subseteq \lambda^{<\omega}$ and a family $d=\left\langle d_{\xi}: \xi<\lambda^{\prime}\right\rangle$ of sets $d_{\xi} \subseteq \lambda^{<\omega}$. We define $T_{d}^{\prime}=\left\{t \in T: \forall \xi<\lambda^{\prime} \exists t^{\prime} \in T \cap d_{\xi}\left(t\right.\right.$ comp $\left.\left.t^{\prime}\right)\right\}$, where $t$ comp $t^{\prime}$ means that $t, t^{\prime}$ are $\subseteq$-comparable, i.e. $t \subseteq t^{\prime}$ or $t^{\prime} \subseteq t$.

The operation can be iterated: we define $T_{d}^{0}=\lambda^{<\omega}, T_{d}^{\alpha+1}=\left(T_{d}^{\alpha}\right)_{d}^{\prime}$ for all ordinals $\alpha$, and $T_{d}^{\gamma}=\bigcap_{\alpha<\gamma} T_{d}^{\alpha}$ for all limit ordinals $\gamma$. There is an ordinal $\varrho$ satisfying $T_{d}^{\varrho}=T_{d}^{\varrho+1}$; let $T_{d}^{\infty}=T_{d}^{\varrho}$. We observe that $T_{d}^{\infty}=\emptyset$ iff there does not exist a branch $a \in \lambda^{\omega}$ such that $\forall \xi<\lambda^{\prime} \exists m\left(a\left\lceil m \in d_{\xi}\right)\right.$.

Consider a weak $\lambda$-Suslin code $\mathrm{d}=\left\langle D_{\xi}: \xi\left\langle\lambda^{\prime}\right\rangle\right.$ for a space $\mathcal{X}=B^{<\omega}$.

For each $x \in X$ and $\xi<\lambda^{\prime}$, let $D_{\xi}(x)=\bigcup_{m}\left\{s \in \lambda^{m}:\langle x \mid m, s\rangle \in D_{\xi}\right\}$. Define $\mathrm{d}(x)=\left\langle D_{\xi}(x): \xi\left\langle\lambda^{\prime}\right\rangle\right.$. Clearly $x \in \mathfrak{p}[\mathrm{d}]$ iff $T_{\mathrm{d}(x)}^{\infty}$ is not empty.

Let $T^{\alpha}(x)=T_{\mathrm{d}(x)}^{\alpha}$, for all $x \in \mathcal{X}$ and $\alpha$. The inner and outer constituents associated with $\mathrm{d}$ are defined, following the standard setup, by

$$
\begin{aligned}
& \mathfrak{C}_{\text {inn }}^{\alpha}(\mathrm{d})=\left\{x \in \mathcal{X}: T^{\alpha}(x)=T^{\alpha+1}(x) \neq \emptyset \& \forall \beta<\alpha\left(T^{\beta}(x) \neq T^{\alpha}(x)\right)\right\} \\
& \mathfrak{C}_{\text {out }}^{\alpha}(\mathrm{d})=\left\{x \in \mathcal{X}: T^{\alpha}(x)=\emptyset \& T^{\beta}(x) \neq \emptyset \text { for all } \beta<\alpha\right\} .
\end{aligned}
$$


Lemma 5. Assume that $\lambda<\omega_{1}$ and $\mathrm{d}$ is a weak $\lambda$-Suslin code. Then $\mathfrak{p}[\mathrm{d}]=\bigcup_{\alpha<\omega_{1}} \mathfrak{C}_{\text {inn }}^{\alpha}(\mathrm{d})$ and $\mathfrak{C p}[\mathrm{d}]=\bigcup_{\alpha<\omega_{1}} \mathfrak{C}_{\text {out }}^{\alpha}(\mathrm{d})$. The constituents $\mathfrak{C}_{\mathrm{inn}}^{\alpha}(\mathrm{d})$ and $\mathfrak{C}_{\text {out }}^{\alpha}(\mathrm{d})$ are Borel sets. If $\mathrm{d} \in \mathrm{L}$ then the constituents $\mathfrak{C}_{\mathrm{inn}}^{\alpha}(\mathrm{d})$ and $\mathfrak{C}_{\text {out }}^{\alpha}(\mathrm{d})$ are $\omega_{1}$-L-Borel sets, even $(\lambda+1)$-L-Borel provided $\alpha<\left(\lambda^{+}\right)^{\mathrm{L}}$.

Proof. The sets $X_{t}^{\alpha}=\left\{x: t \in T^{\alpha}(x)\right\}$ and $\Xi_{\xi t}=\left\{x: t \in D_{\xi}(x)\right\}$ $\left(t \in \lambda^{<\omega}, \xi<\lambda^{\prime}\right.$, and $\left.\alpha<\omega_{1}\right)$ satisfy the following classical equalities:

$$
\begin{aligned}
X_{t}^{0} & =X ; \\
\Xi_{\xi t} & =\left\{x:\langle x \mid m, t\rangle \in D_{\xi}\right\}, \quad \text { where } m=\operatorname{dom} t ; \\
X_{t}^{\alpha+1} & =X_{t}^{\alpha} \cap \bigcap_{\xi<\lambda^{\prime}} \bigcup_{t^{\prime} \text { comp } t}\left(X_{t^{\prime}}^{\alpha} \cap \Xi_{\xi t^{\prime}}\right) ; \\
X_{t}^{\gamma} & =\bigcap_{\alpha<\gamma} X_{t}^{\alpha} \quad \text { for all limit ordinals } \gamma ; \\
\mathfrak{C}_{\text {out }}^{\alpha}(\mathrm{d}) & =\complement X_{\Lambda}^{\alpha} \cap \bigcap_{\beta<\alpha} X_{\Lambda}^{\beta} ; \\
\mathfrak{C}_{\mathrm{inn}}^{\alpha}(\mathrm{d}) & =X_{\Lambda}^{\alpha} \cap \bigcap_{t}\left(X_{t}^{\alpha+1} \cup \complement X_{t}^{\alpha}\right) \cap \bigcap_{\beta<\alpha} \bigcup_{t}\left(X_{t}^{\beta} \backslash X_{t}^{\alpha}\right) .
\end{aligned}
$$

(Here $t$ and $t^{\prime}$ vary over $\lambda^{<\omega}$ while $\alpha<\omega_{1}$.) Since all operations involved are effectively presented we easily obtain $\omega_{1}$-Borel codes for the constituents $\mathfrak{C}_{\text {out }}^{\alpha}(\mathrm{d})$ and $\mathfrak{C}_{\text {inn }}^{\alpha}(\mathrm{d})$ by induction on $\alpha<\omega_{1}$ in L.

Corollary 6. Let $\lambda<\omega_{1}$. Every weak $\lambda$-L-Suslin set, as well as its complement, is a union of $\aleph_{1}$-many $\omega_{1}$-L-Borel sets.

Theorem 7 [Separation]. Let $\lambda<\omega_{1}$. Two disjoint weak $\lambda$-L-Suslin sets can be separated by a $(\lambda+1)$-L-Borel set.

Proof. Consider a pair of weak $\lambda$-Suslin codes $d, d^{\prime} \in$ L. They define the sets $X=\mathfrak{p}[\mathrm{d}]$ and $X^{\prime}=\mathfrak{p}\left[\mathrm{d}^{\prime}\right]$. Assume that $X \cap X^{\prime}=\emptyset$.

Suppose for a moment that $\left(\lambda^{+}\right)^{\mathrm{L}}=\omega_{1}$. In view of Lemma 5 it suffices to prove that $X^{\prime} \subseteq \mathfrak{C}_{\text {out }}^{\alpha}(\mathrm{d})$ for some $\alpha<\omega_{1}$. The proof of this "boundedness" result can be obtained by a routine modification of a classical proof.

(One easily proves that the function $\nu$ sending each $x \in \complement \mathcal{C}$ to the unique $\alpha<\omega_{1}$ such that $x \in \mathfrak{C}_{\text {out }}^{\alpha}(\mathrm{d})$, is a $\boldsymbol{\Pi}_{1}^{1}$-norm, in particular, there is a $\boldsymbol{\Sigma}_{1}^{1}$ relation $P(x, y)$ such that $\nu(x)<\nu(y) \Leftrightarrow P(x, y)$ for all $x, y \in \complement X$. It follows that the relation: $x \prec y$ iff $x, y \in X^{\prime}$ and $\nu(x)<\nu(y)$, is a well-founded $\boldsymbol{\Sigma}_{1}^{1}$ relation on $X^{\prime}$. Hence $\prec$ has a length $\gamma<\omega_{1}$ by the Kunen-Martin theorem. This implies $X^{\prime} \subseteq \mathfrak{C}_{\text {out }}^{\alpha}(\mathrm{d})$ for some $\alpha<\omega_{1}$.)

If $\left(\lambda^{+}\right)^{\mathrm{L}}<\omega_{1}$ then the universe includes a $\lambda$-collapse extension $\mathrm{V}^{\prime}$ of $\mathrm{L}$, so that $\left(\lambda^{+}\right)^{\mathrm{L}}=\omega_{1}$ in $\mathrm{V}^{\prime}$. This allows one to get the result in $\mathrm{V}^{\prime}$, as above, and then extend it to the universe by absoluteness. 
2. Approach to the proof of Theorem 2. Let V, $\kappa \in \mathrm{V}$, and $\mathrm{G}$ denote the universe, the cardinal, and the graph for which Theorem 2 is being proved. Let us fix an ordinal $\lambda, \kappa \leq \lambda<\kappa^{+}$, and a tree $R \subseteq(\omega \times \omega \times \lambda)^{<\omega}$ satisfying $\mathrm{G}=\mathfrak{p}[R]$ in $\mathrm{V}$.

It will be assumed that $R \in \mathrm{L}$. (Otherwise $\mathrm{L}$ uniformly changes to $\mathrm{L}[R]$ throughout the reasoning, including the content of Section 1.)

Let $\mathrm{V}^{+}$be the $\left(\lambda^{++}\right)^{\mathrm{V}}$-collapse generic extension of $\mathrm{V}$.

We are going to define a certain "copy" $\mathrm{G}^{+}$of the graph $\mathrm{G}$ in $\mathrm{V}^{+}$.

If $\mathrm{G}$ is not locally thin in $\mathrm{L}$ then we simply set $\mathrm{G}^{+}=\mathfrak{p}[R]$ in $\mathrm{V}^{+}$, so that $\mathrm{G}^{+}$is the direct $\mathrm{V}^{+}$-copy of $\mathrm{G}$.

Suppose that $\mathrm{G}$ is locally thin in L.

It is a standard fact (see for instance Guaspari [1], Theorem 1.5) that then, in $\mathrm{V}, x \mathrm{G} y \Rightarrow \mathrm{L}[x]=\mathrm{L}[y]$, hence $\mathrm{G} \subseteq \bigcup_{\alpha, \beta<\omega_{1}^{\mathrm{V}}} \mathrm{K}_{\alpha \beta}$ where $\mathrm{K}_{\alpha \beta}$ is the set of all pairs $\langle x, y\rangle \in \mathcal{N}^{2}$ such that $x$ is the $\alpha$ th real in the sense of the Gödel wellordering of $\mathrm{L}[y]$ and $y$ is the $\beta$ th real in the sense of the Gödel wellordering of $\mathrm{L}[x]$.

We define in this case, in $\mathrm{V}^{+}, \mathrm{G}^{+}=\mathfrak{p}[R] \cap \bigcup_{\alpha, \beta<\omega_{1}^{\mathrm{V}}} \mathrm{K}_{\alpha \beta}$.

In both the first and the second case $\mathrm{G}^{+}$is a graph in $\mathrm{V}^{+}$satisfying $\mathrm{G}=$ $\mathrm{G}^{+} \cap \mathrm{V}$. By a simple absoluteness argument $\mathrm{G}^{+}$is disjoint from the diagonal. We can assume that $R$ is symmetric enough for $\mathfrak{p}[R]$ to be symmetric in any model, hence $\mathrm{G}^{+}$is symmetric in $\mathrm{V}^{+}$.

Finally, $\mathrm{G}^{+}$is a weak $\lambda$-L-Suslin set in $\mathrm{V}^{+}$because $\lambda \geq \kappa \geq \omega_{1}^{\mathrm{V}}$.

Definition 8. $\mathcal{C}$ is the collection of all $(\lambda+1)$-Borel codes $C \in \mathrm{L}$ for the space $\mathcal{N}$ such that $[C] \subseteq \mathcal{N}$ is a $\mathrm{G}^{+}$-discrete set in $\mathrm{V}^{+}$.

The following theorem implies Theorem 2 .

Theorem 9. If $\bigcup_{C \in \mathcal{C}}[C]=\mathcal{N}$ in $\mathrm{V}^{+}$then (I) of Theorem 2 holds in $\mathrm{V}$. But if $\cup_{C \in \mathcal{C}}[C] \varsubsetneqq \mathcal{N}$ in $\mathrm{V}^{+}$then (II) of Theorem 2 holds in $\mathrm{V}$.

The first part of Theorem 9 will be proved by a compactness argument: we show in the next section that the assumption leads to a set $\mathcal{C}^{\prime} \subseteq \mathcal{C}$, $\mathcal{C}^{\prime} \in \mathrm{L}$, of cardinality $\leq \lambda$ in $\mathrm{L}$, such that the union $\bigcup_{C \in \mathrm{C}^{\prime}}[C]$ still covers $\mathcal{N}$ in $\mathrm{V}^{+}$, therefore in $\mathrm{V}$ as well, which yields the required colouring.

The second part (getting a homomorphism or embedding, Sections 4 and 5) involves a splitting construction similar to that of Kechris et al. [9].

Proposition 10. The set $\mathcal{C}$ belongs to $\mathrm{L}$.

Proof. As the ordinal $\left(\lambda^{+}\right)^{\mathrm{L}}$ is countable in $\mathrm{V}^{+}$, this universe includes a $\lambda$-collapse extension of $\mathrm{L}$, so, by the Shoenfield theorem, $C \in \mathcal{C}$ iff the $\lambda$-collapse forcing forces over $\mathrm{L}$ that $[C]$ is $\mathrm{G}^{+}$-discrete. 
3. Getting a colouring. Thus we assume that the union $\bigcup_{C \in \mathrm{C}}[C]$ covers the reals in $\mathrm{V}^{+}$.

Lemma 11 [Compactness]. Let $\mathcal{C} \in \mathrm{L}$ be a collection of $(\lambda+1)$-Borel codes for a fixed space of the form $\mathcal{S}=\mathcal{N}^{m} \times\left(\lambda^{\omega}\right)^{n}$ such that $\mathcal{S}=\bigcup_{C \in \mathcal{C}}[C]$ in $\mathrm{V}^{+}$. Then there exists a subcollection $\mathrm{C}^{\prime} \subseteq \mathcal{C}, \mathrm{e}^{\prime} \in \mathrm{L}$, of cardinality $\leq \lambda$ in $\mathrm{L}$, such that we still have $\mathcal{S}=\bigcup_{C \in \mathrm{C}^{\prime}}[C]$ in $\mathrm{V}^{+}$.

Proof $\left({ }^{9}\right)$. Otherwise the set $\mathcal{P}$ of all $(\lambda+1)$-Borel codes $c \in \mathrm{L}$ for $\mathcal{S}$ such that for any set $\mathcal{C}^{\prime} \subseteq \mathcal{C}, \mathcal{C}^{\prime} \in \mathrm{L}$, of cardinality $\leq \lambda$ in $\mathrm{L}$, we have $[c] \nsubseteq \bigcup_{C \in \mathcal{C}^{\prime}}[C]$ in $\mathrm{V}^{+}$, is non-empty. We order $\mathcal{P}$ as follows: $c \leq c^{\prime}\left(c^{\prime}\right.$ is stronger as a forcing condition) iff $\left[c^{\prime}\right] \subseteq[c]$ in $\mathrm{V}^{+}$. Notice that both $\mathcal{P}$ and the order belong to L, as in Proposition 10.

Consider a $\mathcal{P}$-generic (over $\mathrm{V}$ ) set $\mathcal{G} \subseteq \mathcal{P}, \mathcal{G} \in \mathrm{V}^{+}$.

We assert that $\bigcap_{c \in \mathcal{G}}[c] \neq \emptyset$ in $\mathrm{V}^{+}$.

Any point $x \in \bigcap_{c \in \mathcal{G}}[c]$ is then contained in some $[C], C \in \mathcal{C}$, which contradicts the genericity of $\mathcal{G}$, because the set $\mathcal{D}_{C}$ of all codes $c \in \mathcal{P}$ satisfying $[c] \cap[C]=\emptyset$ in $\mathrm{V}^{+}$belongs to $\mathrm{L}$ and is dense in $\mathcal{P}$.

Thus let us prove the emphasized assertion.

This is a standard argument. First, there is, in $\mathrm{V}^{+}$, a unique point $x \in \mathcal{S}$ contained in every basic clopen set the natural code of which belongs to $\mathcal{G}$. We prove that $x \in[c]$ for every code $c \in \mathcal{G}$.

Assume that this is not the case. Consider a code $c=\langle T, F\rangle \in \mathcal{G}$ satisfying $x \notin[c]$ such that the tree $T$ has the least possible rank.

Obviously $T$ cannot be equal to $\{\Lambda\}$. Therefore $[c]=\complement \bigcup_{\nu<\lambda}\left[c_{\nu}\right]$, where $\left\langle c_{\nu}: \nu\langle\lambda\rangle \in \mathrm{L}\right.$ is a sequence of $(\lambda+1)$-Borel codes $c_{\nu}=\left\langle T_{\nu}, F_{\nu}\right\rangle$ having the ranks of the trees $T_{\nu}$ less than the rank of $T$, so $x \in\left[c_{\nu}\right]$ for some $\nu$.

It is impossible, as above, that $T_{\nu}=\{\Lambda\}$, so in fact $\left[c_{\nu}\right]=\complement \bigcup_{\xi<\lambda}\left[c_{\xi}^{\prime}\right]$, where $\left\langle c_{\xi}^{\prime}: \xi<\lambda\right\rangle \in \mathrm{L}$ is a sequence of $(\lambda+1)$-Borel codes $c_{\xi}^{\prime}=\left\langle T_{\xi}^{\prime}, F_{\xi}^{\prime}\right\rangle$ having the ranks of the trees $T_{\xi}^{\prime}$ smaller than the rank of $T_{\nu}$. Since $[c] \subseteq$ $\bigcup_{\xi<\lambda}\left[c_{\xi}^{\prime}\right]$, it follows from the genericity that at least one among the sets $\left[c_{\xi}^{\prime}\right]$ belongs to $\mathcal{G}$. Then $x \in\left[c_{\xi}^{\prime}\right]$, which is a contradiction as $x \in\left[c_{\nu}\right]$.

Coming back to the first part of Theorem 9, we observe that by the lemma there exists a set $\mathrm{C}^{\prime} \subseteq \mathcal{C}, \mathrm{C}^{\prime} \in \mathrm{L}$, of cardinality $\leq \lambda$ in $\mathrm{L}$, such that $\mathcal{N}=\bigcup_{C \in \mathcal{C}^{\prime}}[C]$ in $\mathrm{V}^{+}$. Then $\mathcal{N}=\bigcup_{C \in \mathcal{C}^{\prime}}[C]$ in $\mathrm{V}$ as well. 1 st part of Thm. 9

4. Getting a homomorphism or an embedding. We begin the proof of the second part of Theorem 9 . Thus suppose that the set $\mathfrak{X}=\complement \bigcup_{C \in \mathcal{C}}[C]$ is non-empty in $\mathrm{V}^{+}$.

$\left({ }^{9}\right)$ It is mentioned by the referee that the lemma admits a more straightforward proof which involves countable submodels and an absoluteness argument. 
4.1. Preparations for the locally thin case. We have to take some time in order to prepare consideration of the locally thin case in (II) of Theorem 2. The aim is to get a system of weak Suslin codes for the graphs of the form $\mathrm{G}^{+}{ }_{\alpha \beta}=\mathrm{G}^{+} \cap \mathrm{K}_{\alpha \beta}\left(\alpha, \beta<\omega_{1}^{\mathrm{V}}\right)$ in $\mathrm{V}$.

Lemma 12. Let $\alpha, \beta<\omega_{1}$. Then $\mathrm{K}_{\alpha \beta}$ (see the beginning of Section 2) is a weak $\gamma$-L-Suslin set, where $\gamma=\max \{\alpha, \beta\}$.

Proof. Let wo be the $\Pi_{1}^{1}$ set of all reals $w \in \mathcal{N}$ which code ordinals, and $|w|$ be the ordinal coded by $w \in$ WO. We put $\mathrm{WO}_{\xi}=\{w \in$ WO $:|w|=\xi\}$.

It is a standard fact that there is a $\Sigma_{1}^{1}$ formula $\sigma\left(w, w^{\prime}, x, y\right)$ such that

$$
\forall w \in \mathrm{WO}_{\alpha} \forall w^{\prime} \in \mathrm{WO}_{\beta} \forall x, y \in \mathcal{N}\left(\mathrm{K}_{\alpha \beta}(x, y) \Leftrightarrow \sigma\left(w, w^{\prime}, x, y\right)\right) .
$$

Now, as every set $\mathrm{WO}_{\xi}$ is clearly $(\xi+1)$-L-Borel, hence weak $\gamma$-L-Suslin provided $\xi \leq \gamma$, by Lemma 4 , the set $\mathrm{K}_{\alpha \beta}$ is weak $\gamma$-L-Suslin as well.

Fix a bijection $\gamma \in \mathrm{L}, \gamma: \omega_{1}^{\mathrm{V}} \times \omega_{1}^{\mathrm{V}} \stackrel{\text { onto }}{\longrightarrow} \omega_{1}^{\mathrm{V}}$, satisfying $\gamma(\alpha, \beta) \geq$ $\max \{\alpha, \beta\}$. The proof of the lemma is effective enough to get a sequence $\left\langle\mathrm{q}^{\gamma}: \gamma\left\langle\omega_{1}^{\mathrm{V}}\right\rangle \in \mathrm{L}\right.$ of weak $\lambda$-Suslin codes $\mathrm{q}^{\gamma}=\left\langle Q_{\xi}^{\gamma}: \xi\langle\gamma+\omega\rangle \in \mathrm{L}\right.$ (where $Q_{\xi}^{\gamma} \subseteq(\omega \times \omega \times \lambda)^{<\omega}$ for all $\gamma$ and $\xi$ ) such that $\mathrm{K}_{\alpha \beta}=\mathfrak{p}\left[\mathfrak{q}^{\gamma(\alpha, \beta)}\right]$ for all $\alpha, \beta<\omega_{1}^{\mathrm{V}}$. A suitable enumeration of indices in $\mathrm{V}$ converts this into a sequence of (now not necessarily constructible) weak $\lambda$-Suslin codes $\mathbf{k}^{\gamma}=\left\langle K_{n}^{\gamma}: n \in \omega\right\rangle$ such that $\left[\mathrm{q}^{\gamma}\right]=\left[\mathrm{k}^{\gamma}\right]$, hence $\mathrm{K}_{\alpha \beta}=\mathfrak{p}\left[\mathrm{k}^{\gamma(\alpha, \beta)}\right]$ for all $\alpha, \beta<\omega_{1}^{\mathrm{V}}$, both in $\mathrm{V}$ and in $\mathrm{V}^{+}$. Note that, although possibly $\mathrm{k}^{\gamma} \notin \mathrm{L}$, the set $\left[\mathbf{k}^{\gamma}\right]=\left[\mathbf{q}^{\gamma}\right] \subseteq \mathcal{N}^{2} \times \lambda^{\omega}$ is $(\lambda+1)$-L-Borel (actually $(\lambda+1)$-L-G $\mathbf{G}_{\delta}$ in some sense) for all $\gamma<\omega_{1}^{\mathrm{V}}$, both in $\mathrm{V}$ and in $\mathrm{V}^{+}$.

Let us code intersections of the form $\mathfrak{p}[R] \cap \mathrm{K}_{\alpha \beta}$. Let $\gamma<\omega_{1}^{\mathrm{V}}$. Applying in $\mathrm{V}$ a suitable standard construction, one easily defines a weak $\lambda$-Suslin code $\mathrm{d}^{\gamma}=\left\langle D_{n}^{\gamma}: n \in \omega\right\rangle$ (where $D_{n}^{\gamma} \subseteq(\omega \times \omega \times \lambda)^{<\omega}$ ) such that the set $\left[\mathrm{d}^{\gamma}\right] \subseteq \mathcal{N}^{2} \times \lambda^{\omega}$ is $(\lambda+1)$-L-Borel and $\mathfrak{p}[R] \cap \mathfrak{p}\left[\mathrm{k}^{\gamma}\right]=\mathfrak{p}\left[\mathrm{d}^{\gamma}\right]$ in both $\mathrm{V}$ and $\mathrm{V}^{+}$, in particular

- $\mathrm{G} \cap \mathrm{K}_{\alpha \beta}=\mathfrak{p}\left[\mathrm{d}^{\gamma(\alpha, \beta)}\right]$ in $\mathrm{V}$ and $\mathrm{G}^{+} \cap \mathrm{K}_{\alpha \beta}=\mathfrak{p}\left[\mathrm{d}^{\gamma(\alpha, \beta)}\right]$ in $\mathrm{V}^{+}$.

We can assume without any loss of generality that $D_{n+1}^{\gamma} \subseteq D_{n}^{\gamma}$ for all $\gamma, n$.

If $\mathrm{G}$ is not locally thin (in $\mathrm{V}$ ) then the construction above is needless but, to keep the integrity of the reasoning, we define, in $\mathrm{V}$, for each ordinal $\gamma<\omega_{1}^{\mathrm{V}}$, a weak $\lambda$-Suslin code $\mathrm{d}^{\gamma}=\left\langle D_{n}^{\gamma}: n \in \omega\right\rangle \in \mathrm{L}$ by

$$
D_{n}^{\gamma}=\left\{\langle u, v, r\rangle \in R: \operatorname{dom} u=\operatorname{dom} v=\operatorname{dom} r=n^{\prime} \geq n\right\}
$$

so that $\mathfrak{p}\left[\mathrm{d}^{\gamma}\right]=\mathfrak{p}[R]$ in both $\mathrm{V}$ and $\mathrm{V}^{+}$, in particular $\mathfrak{p}\left[\mathrm{d}^{\gamma}\right]=\mathrm{G}$ in $\mathrm{V}$ and (in the case when $\mathrm{G}$ is not locally thin) $\mathfrak{p}\left[\mathrm{d}^{\gamma}\right]=\mathrm{G}^{+}$in $\mathrm{V}^{+}$.

We observe that $\mathrm{G}^{+}=\bigcup_{\gamma<\omega_{1}^{\mathrm{v}}} \mathfrak{p}\left[\mathrm{d}^{\gamma}\right]$ in any of the two cases. 
4.2. Crucial pairs. Recall that a sequence of binary tuples $\mathfrak{s}_{m} \in 2^{m}$ is fixed by the definition of $\mathrm{G}_{0}$ (see Introduction). Let $m \in \omega$. By a crucial pair in $2^{m}$ we shall understand any pair $\langle u, v\rangle$ of tuples $u, v \in 2^{m}$ such that $u=\mathfrak{s}_{k} \wedge 0^{\wedge} w$ and $v=\mathfrak{s}_{k} \wedge 1^{\wedge} w$, for some $k<m$ and $w \in 2^{m-k-1}$. (Possibly $w=\Lambda$, the empty sequence.) Let $\mathrm{CP}_{m}$ denote the set of all crucial pairs in $2^{m}$.

Note that crucial pairs in $2^{m+1}$ are pairs of the form $\left\langle u^{\wedge} i, v^{\wedge} i\right\rangle$ where $\langle u, v\rangle$ is a crucial pair in $2^{m}$ and $i=0,1$, plus the pair $\left\langle\mathfrak{s}_{m} \wedge 0, \mathfrak{s}_{m} \wedge 1\right\rangle$. Moreover, the (directed) graph of crucial pairs in $2^{m}$ is a tree: each pair of $u, v \in 2^{m}$ is connected in $2^{m}$ by a unique (non-self-intersecting) chain of crucial pairs $u=u_{0} \sim u_{1} \sim u_{2} \sim \ldots \sim u_{n-1} \sim u_{n}=v$, where $u^{\prime} \sim v^{\prime}$ means that either $\left\langle u^{\prime}, v^{\prime}\right\rangle$ or $\left\langle v^{\prime}, u^{\prime}\right\rangle$ is a crucial pair. (The property of being uniquely connected by crucial pairs is inherited from $2^{m}$ by each of the two parts, $H_{0}=\left\{u^{\wedge} 0: u \in 2^{m}\right\}$ and $H_{1}=\left\{u^{\wedge} 1: u \in 2^{m}\right\}$, of $2^{m+1}$, and there is a unique crucial pair, namely $\left\langle\mathfrak{s}_{m} \wedge 0, \mathfrak{s}_{m} \wedge 1\right\rangle$, connecting the parts.)

4.3. Splitting systems. We shall define, in $\mathrm{V}$, a system of finite sequences $\tau_{u} \in \omega^{<\omega}\left(u \in 2^{<\omega}\right)$ and $\sigma_{u v} \in \lambda^{<\omega}\left(\langle u, v\rangle \in \mathrm{CP}_{m}\right.$ for some $\left.m\right)$, and an ordinal $\gamma_{k}<\omega_{1}^{\mathrm{V}}$ for each $k \in \omega$, satisfying the following four requirements for all $m$ :

(i) $\tau_{u} \subset \tau_{u^{\wedge} i}$ for all $u \in 2^{m}$ and $i=0,1$, and $\sigma_{u v} \subset \sigma_{u^{\wedge} i, v^{\wedge} i}$ for all $\langle u, v\rangle \in \mathrm{CP}_{m}$ and $i=0,1$;

(ii) if $\langle u, v\rangle \in \mathrm{CP}_{m}, k<m$, and $u=\mathfrak{s}_{k}{ }^{\wedge} 0^{\wedge} w, v=\mathfrak{s}_{k}{ }^{\wedge} 1^{\wedge} w$ for some $w \in 2^{m-k-1}$, then $\left\langle\tau_{u}, \tau_{v}, \sigma_{u v}\right\rangle \in D_{m}^{\gamma_{k}}$;

(iii) there exists, in $\mathrm{V}^{+}$, a system of reals $x_{u} \in \mathcal{N}_{\tau_{u}} \cap \mathfrak{X}\left(u \in 2^{m}\right)$ and functions $f_{u v} \in \lambda^{\omega}\left(\langle u, v\rangle \in \mathrm{CP}_{m}\right)$ such that, for any $k<m$ and any pair $\langle u, v\rangle \in \mathrm{CP}_{m}$ of the form $u=\mathfrak{s}_{k}{ }^{\wedge} 0^{\wedge} w, v=\mathfrak{s}_{k}{ }^{\wedge} 1^{\wedge} w$, for some $w \in 2^{m-k-1}$, we have $\sigma_{u v} \subset f_{u v}$ and $\left\langle x_{u}, x_{v}, f_{u v}\right\rangle \in\left[\mathrm{d}^{\gamma_{k}}\right]$;

(iv) if $\mathrm{G}$ is either acyclic or locally thin in $\mathrm{V}, u, v \in 2^{m}$, and $u \neq v$, then $\tau_{u}$ and $\tau_{v}$ are incomparable in $\omega^{<\omega}$.

Requirement (iii) is formally expressed in $\mathrm{V}^{+}$, but, as the existence of the reals and functions which witness (iii) is suitably forced even in L (see the proof of Proposition 10), we are essentially still in V.

Having such a system, we set, in $\mathrm{V}, H(a)=\bigcup_{m \in \omega} \tau_{a \uparrow m}$ for any $a \in 2^{\omega}$, so that $H: 2^{\omega} \rightarrow \mathcal{N}$ is a continuous map. We prove that $\mathrm{G}_{0} \leq_{\mathrm{c}} \mathrm{G}$ in V via $H$. Suppose that $a, b \in 2^{\omega}$ in $\mathrm{V}$ and $a \mathrm{G}_{0} b$. By definition we have e.g. $a=\mathfrak{s}_{k}{ }^{\wedge} 0^{\wedge} c$ and $b=\mathfrak{s}_{k} \wedge 1^{\wedge} c$ for some $k$ and $c \in 2^{\omega}$. Then, by (ii),

$$
\left\langle\tau_{\mathfrak{s}_{k} \wedge 0^{\wedge}(c \mid n)}, \tau_{\mathfrak{s}_{k} \wedge 1^{\wedge}(c \mid n)}, \sigma_{\mathfrak{s}_{k} \wedge} 0^{\wedge}(c \mid n), \mathfrak{s}_{k} \wedge 1^{\wedge}(c \mid n)\right\rangle \in D_{n+k+1}^{\gamma_{k}}
$$

for all $n \in \omega$. It easily follows that $\langle H(a), H(b)\rangle \in \mathfrak{p}\left[\mathrm{d}^{\gamma_{k}}\right] \subseteq \mathrm{G}$ as required.

Moreover, it follows from (iv) that $H$ is a 1-1 map, i.e. an embedding, provided $\mathrm{G}$ is acyclic or locally thin in $\mathrm{V}$. 
Thus the proof of the second part of Theorem 9 has been reduced to the construction of a splitting system satisfying (i)-(iv).

5. Construction of a splitting system. Let, for any $m, S_{m}$ be the collection of all finite systems of tuples $\tau_{u}$ (where $\left.u \in 2^{\leq m}\right)$ and $\sigma_{u v}(\langle u, v\rangle \in$ $\mathrm{CP}_{n}$ and $\left.n \leq m\right)$ and ordinals $\gamma_{k}(k<m$ strictly) satisfying the requirements at the level $m$ and below.

(For instance, setting $\tau_{\Lambda}=\Lambda$, we obtain a system in $S_{0}$.)

We observe that each set $S_{m}$ and the whole sequence of them belong to V (as in Proposition 10 above).

Lemma 13. Any system in $S_{m}$ can be expanded to a system in $S_{m+1}$.

Proof. Consider a system of tuples $\tau_{u} \in \omega^{<\omega}$ and $\sigma_{u v} \in \lambda^{<\omega}$ and ordinals $\gamma_{k}$ which belongs to $S_{m}$. We define an expansion at the level $m+1$.

To begin with we define $\tau_{u^{\wedge} i}=\tau_{u}$ for any $u \in 2^{m}, \sigma_{u^{\wedge} i, v^{\wedge} i}=\sigma_{u v}$ for any pair $\langle u, v\rangle \in \mathrm{CP}_{m}$, and all $i=0,1$, and finally $\sigma_{\mathfrak{s}_{m} \wedge 0, \mathfrak{s}_{m} \wedge 1}=\Lambda$. This may not be a correct expansion, and will be later changed.

Note that the ordinal $\gamma_{m}$ has not yet been defined.

AsSERTION 14. In $\mathrm{V}^{+}$, there exist: an ordinal $\gamma_{m}<\omega_{1}^{\mathrm{V}}$, reals $x_{s}$ $\left(s \in 2^{m+1}\right)$, and functions $f_{s t}\left(\langle s, t\rangle \in \mathrm{CP}_{m+1}\right)$ satisfying (iii) at the level $m+1$.

AsSERTION 15. If $\mathrm{G}$ is acyclic or locally thin in $\mathrm{V}$ and, in $\mathrm{V}^{+}$, reals $x_{s}\left(s \in 2^{m+1}\right)$ and functions $f_{s t}\left(\langle s, t\rangle \in \mathrm{CP}_{m+1}\right)$ satisfy (iii) at the level $m+1$ with some $\gamma_{m}<\omega_{1}^{\mathrm{V}}$, then $x_{s} \neq x_{t}$ for all $s \neq t \in 2^{m+1}$.

This easily implies the lemma: indeed, put $u_{s}=x_{s}\left\lceil N\right.$ for all $s \in 2^{m+1}$ and $\sigma_{s t}=f_{s t}\left\lceil N\right.$ for any pair $\langle s, t\rangle \in \mathrm{CP}_{m+1}$, for a large enough natural $N$.

Proof of Assertion 14. Suppose otherwise.

Then, in $\mathrm{V}^{+}$, for each $\gamma<\omega_{1}^{\mathrm{V}}$ the collection $\mathbf{X}_{\gamma}$ of all systems of reals $x_{s} \in \mathcal{N}_{s} \cap \mathfrak{X}\left(s \in 2^{m+1}\right)$ and functions $f_{s t} \in \lambda^{\omega}\left(\langle s, t\rangle \in \mathrm{CP}_{m+1}\right)$ which witness (iii) at the level $m+1$ with $\gamma_{m}=\gamma$ is empty.

Since each $\mathbf{X}_{\gamma}$ is by definition an intersection of sets defined by a (constructible) family of $(\lambda+1)$-Borel codes (including finitely many codes of the $(\lambda+1)$-L-Borel sets $\left.\left[\mathrm{d}^{\gamma_{k}}\right], k<m\right)$, Lemma 11 provides subcollections, of cardinality $\leq \lambda$ in $\mathrm{L}$, such that the intersection still remains empty.

More exactly, there is a set $\mathcal{C}^{\prime} \subseteq \mathcal{C}, \mathcal{C}^{\prime} \in \mathrm{L}$, of cardinality $\leq \lambda$ in $\mathrm{L}$, such that every set $\mathbf{X}_{\gamma}^{\prime}, \gamma<\omega_{1}^{\mathrm{V}}$, defined in $\mathrm{V}^{+}$similarly to $\mathbf{X}_{\gamma}$ but using the bigger set $\mathfrak{X}^{\prime}=\complement \bigcup_{C \in \mathfrak{C}^{\prime}}[C]$ instead of $\mathfrak{X}=\complement \bigcup_{C \in \mathcal{C}}[C]$, is still empty.

Consider, in $\mathrm{V}^{+}$, the set $\mathbf{Y}^{\prime}$ of all systems of reals $y_{u} \in \mathcal{N}_{\tau_{u}} \cap \mathfrak{X}^{\prime}\left(u \in 2^{m}\right)$ and functions $f_{u v} \in \lambda^{\omega}\left(\langle u, v\rangle \in \mathrm{CP}_{m}\right)$ which witness (iii) at the level $m$. Note that $\mathbf{Y}^{\prime} \neq \emptyset$ as (iii) is supposed to hold at the level $m$. 
Clearly $\mathbf{Y}^{\prime}$ is a $(\lambda+1)$-L-Borel set. Therefore the set $A$ of all reals $y_{\mathfrak{s}_{m}}$ that occur in $\mathbf{Y}^{\prime}$ (the projection of $\mathbf{Y}^{\prime}$ onto the $\mathfrak{s}_{m}$ th coordinate in $\mathrm{V}^{+}$) is a weak $\lambda$-L-Suslin set in $\mathrm{V}^{+}$by Lemma 4 .

We observe that $A$ is a $\mathrm{G}^{+}$-discrete set in $\mathrm{V}^{+}$. (Indeed, suppose that $y, y^{\prime} \in A$ and $y \mathrm{G}^{+} y^{\prime}$ in $\mathrm{V}^{+}$. As by definition $\mathrm{G}^{+}=\bigcup_{\gamma<\omega_{1}^{v}} \mathfrak{p}\left[\mathrm{d}^{\gamma}\right]$ in $\mathrm{V}^{+}$, there exists an ordinal $\gamma<\omega_{1}^{\mathrm{V}}$ satisfying $\left\langle y, y^{\prime}\right\rangle \in \mathfrak{p}\left[\mathbf{d}^{\gamma}\right]$. This immediately implies $\mathbf{X}_{\gamma} \neq \emptyset$, which contradicts the above.)

Let us cover $A$ by a $(\lambda+1)$-L-Borel $\mathrm{G}^{+}$-discrete set.

Note that $A \subseteq B=\left\{x \in \mathcal{N}: \forall y \in A\left(\neg x \mathrm{G}^{+} y\right)\right\}$ in $\mathrm{V}^{+}$by the discreteness. Clearly $B$ is a weak co- $\lambda$-L-Suslin set in $\mathrm{V}^{+}$. Hence by Theorem 7 there is a $(\lambda+1)$-L-Borel set $D^{\prime}$ such that $A \subseteq D^{\prime} \subseteq B$ in $\mathrm{V}^{+}$.

Now put $A^{\prime}=\left\{x \in D^{\prime}: \forall y \in D^{\prime}\left(\neg x \mathrm{G}^{+} y\right)\right\}$, so $A \subseteq A^{\prime} \subseteq D^{\prime}$ in $\mathrm{V}^{+}$. The same argument yields a $(\lambda+1)$-L-Borel set $D$ such that $A \subseteq D \subseteq A^{\prime}$ in $\mathrm{V}^{+}$. We now observe that $D$ is a $\mathrm{G}^{+}$-discrete set in $\mathrm{V}^{+}$by the construction, hence $D=[C]$ for a code $C \in \mathcal{C}$.

As (iii) is satisfied at the level $m$, there exists, in $\mathrm{V}^{+}$, a system of reals $y_{u} \in \mathfrak{X} \cap \mathcal{N}_{\tau_{u}}\left(u \in 2^{m}\right)$ and functions $f_{u v} \in \lambda^{\omega}\left(\langle u, v\rangle \in \mathrm{CP}_{m}\right)$ satisfying (iii) at the level $m$. The system belongs to $\mathbf{Y}^{\prime}$ because $\mathfrak{X} \subseteq \mathfrak{X}^{\prime}$. Thus $y=$ $y_{\mathfrak{s}_{m}} \in A \subseteq[C]$, which is a contradiction since $\mathfrak{X}=\complement \bigcup_{C \in \mathcal{C}}[C]$.

Proof of Assertion 15. As (iv) is supposed to hold at the level $m$, we have to prove only the following: $x_{u^{\wedge} 0} \neq x_{u^{\wedge} 1}$ for all $u \in 2^{m}$.

Acyclic case. Note that the acyclicity of a Suslin graph is an absolute property. (The absence of a cycle of a length $n \geq 3$ can be expressed as the well-foundedness of a certain tree derived from the given tree.) Therefore $\mathrm{G}^{+}$is acyclic in $\mathrm{V}^{+}$.

However, as the reals $x_{s}$ satisfy (iii), we have $x_{s} \mathrm{G}^{+} x_{t}$ in $\mathrm{V}^{+}$whenever $\langle s, t\rangle \in \mathrm{CP}_{m+1}$. This implies $x_{u^{\wedge} 0} \neq x_{u^{\wedge} 1}$ because $u^{\wedge} 0$ is connected with $u^{\wedge} 1$ in $2^{m+1}$ by a (unique non-self-intersecting) chain of crucial pairs which definitely cannot contain exactly 2 edges.

Locally thin case. There is a unique chain of crucial pairs $\mathfrak{s}_{m}=v_{1} \sim v_{2} \sim$ $\ldots \sim v_{n}=u$ in $2^{m}$. We prove by induction on $l$ that $x_{v_{l} \wedge} \neq x_{v_{l} \wedge 1}$.

The result for $l=1$ holds because $\left\langle x_{\mathfrak{s}_{m} \wedge}, x_{\mathfrak{s}_{m} \wedge}\right\rangle \in \mathfrak{p}\left[\mathrm{d}^{\gamma_{m}}\right] \subseteq \mathrm{G}^{+}$, but by absoluteness $\mathrm{G}^{+}$is disjoint from the diagonal in $\mathrm{V}^{+}$.

We suppose that $x_{v_{l} \wedge 0} \neq x_{v_{l} \wedge 1}$ and prove $x_{v_{l+1} \wedge 0} \neq x_{v_{l+1} \wedge 1}$. One of the pairs $\left\langle v_{l}, v_{l+1}\right\rangle$ and $\left\langle v_{l+1}, v_{l}\right\rangle$ is crucial; suppose that this is the first one. Then, as (iii) is satisfied, there is an ordinal $\gamma<\omega_{1}^{\mathrm{V}}\left(\gamma=\gamma_{k}\right.$ for some $\left.k<m\right)$ such that each of the pairs $\left\langle x_{v_{l} \wedge i}, x_{v_{l+1} \wedge i}\right\rangle, i=0,1$, belongs to $\mathfrak{p}\left[\mathrm{d}^{\gamma}\right]$. However, in the locally thin case $\mathfrak{p}\left[\mathrm{d}^{\gamma}\right]=\mathrm{G}^{+} \cap \mathrm{K}_{\alpha \beta}$ is the graph of a 1-1 function in $\mathrm{V}^{+}$. This implies $x_{v_{l+1} \wedge} \neq x_{v_{l+1} \wedge 1}$ as required. Assert. 15, Lemma 13, Thms. 9, 2 
6. Dichotomy in the Solovay model. This section proves Theorem 3. The proof presented here differs from the proof in Kanovei [7]. We shall simply indicate how the arguments involved in the proof of Theorem 2 above change in the new setting.

By the Solovay model we shall understand a certain generic extension of a constructible model, as defined by Solovay [10].

It is not worthwhile to present here the construction of the model in detail because all that is necessary to prove Theorem 3 is concentrated in the next proposition.

In the remainder, a weak $<\omega_{1}$-Suslin code means a weak $\lambda$-Suslin code for some $\lambda<\omega_{1}$, and a weak $<\omega_{1}-\mathrm{L}\left[z_{0}\right]$-Suslin set means a set of the form $\mathfrak{p}[\mathrm{d}]$ where $\mathrm{d} \in \mathrm{L}\left[z_{0}\right]$ is a weak $<\omega_{1}$-Suslin code.

Proposition 16. The following is true in the Solovay model. Let $z_{0} \in \mathcal{N}$. Then

(1) $\omega_{1}$ is inaccessible in $\mathrm{L}\left[z_{0}\right]$.

(2) If $X \subseteq \mathcal{N}$ is $\mathrm{OD}\left[z_{0}\right]$ and thin then $X \subseteq \mathrm{L}\left[z_{0}\right]$.

(3) If $X \subseteq \mathrm{L}\left[z_{0}\right]$ is $\mathrm{OD}\left[z_{0}\right]$ then $X \in \mathrm{L}\left[z_{0}\right]$.

(4) Every $\mathrm{OD}\left[z_{0}\right]$ set $X \subseteq \mathcal{N}$ is a union of weak $<\omega_{1}-\mathrm{L}\left[z_{0}\right]$-Suslin sets.

Pr o o f. (1)-(3) are standard. (4) is also a rather known fact. We refer to Proposition 5(3) in Kanovei [6]; sets of the form $X_{t}(S)$ in [6] are by definition weak $<\omega_{1}-\mathrm{L}\left[z_{0}\right]-$ Suslin sets provided $S=z_{0}$.

We argue in the Solovay model. Starting the proof of Theorem 3, in order to eliminate the real parameter, let us assume that $\mathrm{G}$ is an $\mathrm{OD}$ graph.

Let us prove that statements (I) and (II) of Theorem 3 are incompatible (in the Solovay model). Suppose on the contrary that they are compatible. Then the graph $\mathrm{G}_{0}$ admits a ROD colouring $\phi: \mathcal{D}=2^{\omega} \rightarrow \omega_{1}$. We obtain a contradiction following an argument in [9]. By known properties of the Solovay model at least one of the sets $X_{\alpha}=\phi^{-1}(\alpha), \alpha<\omega_{1}$, is not meager. Then $X_{\alpha}$ is co-meager on a set of the form $\mathcal{D}_{u}=\{x \in \mathcal{D}: u \subset x\}$, where $u \in 2^{<\omega}$. We have $u \subseteq \mathfrak{s}_{n}$ for some $n$ (see Introduction). Then $X_{\alpha}$ is co-meager on $\mathcal{D}_{\mathfrak{s}_{n}}$. Define an automorphism $H$ of $\mathcal{D}$ by $H(x)=y$, where $y(k)=x(k)$ whenever $k \neq n$, but $y(n)=1-x(n)$. Then $Y=H^{-1}\left(X_{\alpha} \cap \mathcal{D}_{\mathfrak{s}_{n}}\right)$ is co-meager on $\mathcal{D}_{\mathfrak{s}_{n}}$. Hence there is $x \in X_{\alpha} \cap Y \cap \mathcal{D}_{\mathfrak{s}_{n}}$. Now $y=H(x) \in X_{\alpha}$, but $x \mathrm{G}_{0} y$, which is a contradiction.

Let us describe the splitting point of the dichotomy. In principle it has a resemblance with the case $\lambda=\omega_{1}^{\mathrm{V}}$ in the proof of Theorem 2. However, as the Solovay model is already quite generic, we are not in need of any further generic extension, as in Section 2, and Lemma 11 is also needless. 
We define $\mathcal{C}$ to be the set of all $\omega_{1}$-Borel codes $C \in \mathrm{L}$ for the space $\mathcal{N}$ such that $[C]$ is a $\mathrm{G}$-discrete set. Since the case when $\mathcal{N}=\bigcup_{C \in \mathrm{C}}[C]$ easily leads to (I) of Theorem 3, it suffices to prove the following

Theorem 17 (in the Solovay model). If the set $X=\complement \bigcup_{C \in \mathcal{C}}[C]$ is nonempty then (II) of Theorem 3 holds.

Proof. Enumerate, as $\left\langle\mathrm{G}^{\gamma}: \gamma<\omega_{1}\right\rangle$, the set of all weak $<\omega_{1}$-L-Suslin subsets of $G$-in the case when $G$ is not locally thin, and all weak $<\omega_{1}$-L-Suslin subsets of $\mathrm{G}$ which are graphs of (partial) 1-1 functions-in the case when $G$ is locally thin. It follows from Proposition 16((2), (4)) that $\mathrm{G}=\bigcup_{\gamma<\omega_{1}} \mathrm{G}^{\gamma}$ in each of the two cases.

As every $<\omega_{1}$-L-Suslin set is $\omega_{1}$-L-Suslin as well, there exists a sequence $\left\langle\mathrm{d}^{\gamma}: \gamma\left\langle\omega_{1}\right\rangle\right.$ of weak $\omega_{1}$-Suslin codes $\mathrm{d}^{\gamma}=\left\langle D_{m}^{\gamma}: m \in \omega\right\rangle$ (each $D_{m}^{\gamma}$ being a subset of $\left.\left(\omega \times \omega \times \omega_{1}\right)^{<\omega}\right)$ such that $\mathrm{G}^{\gamma}=\mathfrak{p}\left[\mathrm{d}^{\gamma}\right]$ for all $\gamma<\omega_{1}$.

We define, in the Solovay model, a system of finite sequences $\tau_{u} \in \omega^{<\omega}$ $\left(u \in 2^{<\omega}\right)$ and $\sigma_{u v} \in \omega_{1}<\omega\left(\langle u, v\rangle \in \mathrm{CP}_{m}, m \in \omega\right)$, and an ordinal $\gamma_{k}<\omega_{1}$ for each $k \in \omega$, satisfying the following requirements for all $m$ :

(i) $\tau_{u} \subset \tau_{u^{\wedge} i}$ for all $u \in 2^{m}$ and $i=0,1$, and $\sigma_{u v} \subset \sigma_{u^{\wedge} i, v^{\wedge} i}$ for all $\langle u, v\rangle \in \mathrm{CP}_{m}$ and $i=0,1$;

(ii) if $\langle u, v\rangle \in \mathrm{CP}_{m}, k<m$, and $u=\mathfrak{s}_{k}{ }^{\wedge} 0^{\wedge} w, v=\mathfrak{s}_{k}{ }^{\wedge} 1^{\wedge} w$ for some $w \in 2^{m-k-1}$, then $\left\langle\tau_{u}, \tau_{v}, \sigma_{u v}\right\rangle \in D_{m}^{\gamma_{k}}$;

(iii) there exists a system of reals $x_{u} \in \mathcal{N}_{\tau_{u}} \cap \mathfrak{X}\left(u \in 2^{m}\right)$ and functions $f_{u v} \in \omega_{1}^{\omega}\left(\langle u, v\rangle \in \mathrm{CP}_{m}\right)$ such that, for any $k<m$ and any pair $\langle u, v\rangle \in \mathrm{CP}_{m}$ of the form $u=\mathfrak{s}_{k}{ }^{\wedge} 0^{\wedge} w, v=\mathfrak{s}_{k}{ }^{\wedge} 1^{\wedge} w$ for some $w \in 2^{m-k-1}$, we have $\sigma_{u v} \subset f_{u v}$ and $\left\langle x_{u}, x_{v}, f_{u v}\right\rangle \in\left[\mathrm{d}^{\gamma_{k}}\right]$;

(iv) if $\mathrm{G}$ is either acyclic or locally thin, $u, v \in 2^{m}$, and $u \neq v$, then $\tau_{u}$ and $\tau_{v}$ are incomparable in $\omega^{<\omega}$.

(The only difference with (i)-(iii) in Section 4 is that the reals and functions in (iii) are required to exist in the same universe rather than in its generic extension.) Such a system guarantees that $G_{0} \leq_{c} G$, even $G_{0} \sqsubseteq_{c} G$ provided $G$ is acyclic or locally thin, as in Section 4 , so it suffices to carry out the construction satisfying (i)-(iv).

Suppose that the construction has been accomplished at a level $m$, and show how to expand it at the level $m+1$. Following the line of reasoning in Section 4, we first define $\tau_{u^{\wedge} i}=\tau_{u}$ for all $u \in 2^{m}$ and $\sigma_{u^{\wedge} i, v^{\wedge} i}=\sigma_{u v}$ for any pair $\langle u, v\rangle \in \mathrm{CP}_{m}$, and all $i=0,1$, and finally $\sigma_{\mathfrak{s}_{m} \wedge 0, \mathfrak{s}_{m} \wedge 1}=\Lambda$.

Now the problem is to prove Assertions 14 and 15 in Section 5 in the new setting. The proof of Assertion 15 is completely similar to the reasoning in Section 5 (is even simpler because all the time one argues in the Solovay model). Assertion 14 needs a more careful analysis. 
Assertion 18 (A counterpart of Assertion 14 in the Solovay model). There exist an ordinal $\gamma_{m}<\omega_{1}$, reals $x_{s}\left(s \in 2^{m+1}\right)$ and functions $f_{s t} \in \omega_{1}^{\omega}$ $\left(\langle s, t\rangle \in \mathrm{CP}_{m+1}\right)$ satisfying (iii) at the level $m+1$.

Proof. Otherwise for each $\gamma<\omega_{1}$ the collection $\mathbf{X}_{\gamma}$ of all systems of reals $x_{s} \in \mathcal{N}_{s} \cap \mathfrak{X}\left(s \in 2^{m+1}\right)$ and functions $f_{s t} \in \omega_{1}^{\omega}\left(\langle s, t\rangle \in \mathrm{CP}_{m+1}\right)$ satisfying (iii) at the level $m+1$ with $\gamma_{m}=\gamma$ is empty.

However, the set $\mathbf{Y}$ of all systems of reals $y_{u} \in \mathcal{N}_{\tau_{u}} \cap \mathfrak{X}\left(u \in 2^{m}\right)$ and functions $f_{u v} \in \omega_{1}^{\omega}\left(\langle u, v\rangle \in \mathrm{CP}_{m}\right)$ which satisfy (iii) at the level $m$ is non-empty as (iii) is supposed to hold at the level $m$.

Note that the set $A$ of all reals $y_{\mathfrak{s}_{m}}$ that occur in $\mathbf{Y}$ (the projection of $\mathbf{Y}$ onto the $\mathfrak{s}_{m}$ th coordinate) is $\mathrm{G}$-discrete. (Otherwise it is not $\mathrm{G}^{\gamma}$-discrete for some $\gamma<\omega_{1}$ because $\mathrm{G}=\bigcup_{\gamma<\omega_{1}} \mathrm{G}^{\gamma}$, which implies $\mathbf{X}_{\gamma} \neq \emptyset$.)

Consider a system of reals $y_{u} \in \mathcal{N}_{\tau_{u}} \cap \mathfrak{X}\left(u \in 2^{m}\right)$ and functions $f_{u v} \in \omega_{1}^{\omega}$ $\left(\langle u, v\rangle \in \mathrm{CP}_{m}\right)$ which belongs to $\mathbf{Y}$. Then $y_{\mathfrak{s}_{m}} \in A$.

By Proposition 16, $A$ is the union of weak $<\omega_{1}$-L-Suslin sets $A^{\prime} \subseteq A$. (This is the point where we avoid the involvement of a generic extension of the universe. Any set is "enough Suslin" in the Solovay model.) Moreover, it follows from Corollary $6\left({ }^{10}\right)$ that $A$ is the union of all $\omega_{1}$-L-Borel sets $B \subseteq A$. Therefore $y_{\mathfrak{s}_{m}}$ belongs to an $\omega_{1}$-L-Borel set $B \subseteq A$ which is G-discrete together with $A$. Thus $y_{\mathfrak{s}_{m}} \notin \mathfrak{X}$, which is a contradiction.

-Assert. 18, Thms. 17, 3

7. A simplified dichotomy for $\Sigma_{2}^{1}$ graphs. This section presents a short proof of the fact that any $\boldsymbol{\Sigma}_{2}^{1}$ graph $G$ satisfies $\chi(G) \leq \aleph_{1}$ or $G_{0} \leq_{c} G$. The proof reduces the problem to Theorem 1 via an argument similar to a proof of the Burgess theorem on the number of equivalence classes of $\boldsymbol{\Sigma}_{1}^{1}$ equivalence relations from the Silver theorem on $\boldsymbol{\Pi}_{1}^{1}$ relations, as described by Hjorth [4].

We may suppose, as usual, that $\mathrm{G}$ is a $\Sigma_{2}^{1}$ graph. It is a classical theorem that $\mathrm{G}$ has the form of a union $\mathrm{G}=\bigcup_{\gamma<\omega_{1}} \mathrm{G}^{\gamma}$ of Borel subgraphs $\mathrm{G}^{\gamma} \subseteq \mathrm{G}$. Such a decomposition can be realized in such a way that the statement: "there exist an ordinal $\gamma<\omega_{1}$ and a continuous function $H$ which witnesses $\mathrm{G}_{0} \leq_{\mathrm{c}}\left(\bigcup_{\alpha<\gamma} \mathrm{G}^{\alpha}\right)$ " is $\Sigma_{2}^{1}$, therefore absolute.

Let, as above, $\mathrm{V}$ be the universe in which we prove the result.

We set $\kappa=\omega_{1}^{\mathrm{V}}$. Consider a $\kappa$-collapse extension $\mathrm{V}^{+}$of $\mathrm{V}$. Let $\mathrm{G}^{+}$be the $\mathrm{V}^{+}$-copy of $\mathrm{G}$ defined in $\mathrm{V}^{+}$by the same $\Sigma_{2}^{1}$ formula. Let finally $\Gamma \in \mathrm{L}$ be a $(\kappa+1)$-Borel code which naturally defines the approximation $\bigcup_{\alpha<\kappa} \mathrm{G}^{\alpha}$ of $\mathrm{G}$, so that $\mathrm{G}=[\Gamma]$ in $\mathrm{V}$, but perhaps $[\Gamma] \varsubsetneqq \mathrm{G}^{+}$in $\mathrm{V}^{+}$.

$\left({ }^{10}\right)$ This argument might also have been used in the proof of Assertion 14, instead of a sophisticated application of the separation theorem; however, this would lead to a somewhat weaker result than Theorem 2 . 
Then $[\Gamma]$ is a Borel graph in $\mathrm{V}^{+}$. It follows from Theorem 1 that, in $\mathrm{V}^{+}$, either $[\Gamma]$ is countably Borel colourable or $\mathrm{G}_{0} \leq_{\mathrm{c}}[\Gamma]$. In the "or" case, we immediately obtain $\mathrm{G}_{0} \leq_{\mathrm{c}} \mathrm{G}$ in $\mathrm{V}$ by the absoluteness argument above.

Thus assume that $[\Gamma]$ is countably Borel colourable in $\mathrm{V}^{+}$.

Let $P$ be the $\kappa$-collapse forcing; in particular, $P \in \mathrm{L}$ is a p.o. set of cardinality $\leq \kappa$ in L. Let us fix a P-term $\mathfrak{t}$ such that, in $\mathrm{V}, P$ forces $\mathfrak{t}$ to be a colouring of $[\Gamma]$ by natural numbers.

We define, in $\mathrm{V}$, for any real $x, h(x)$ to be the least, e.g. in the sense of the Gödel wellordering of $\mathrm{L}$, pair $\langle p, n\rangle \in P \times \omega$ such that $p$ forces $\mathfrak{t}(x)=n$. Clearly $h$ witnesses that $\chi(\mathrm{G}) \leq \aleph_{1}$ in $\mathrm{V}$.

This argument can be elaborated, towards a stronger result: if $\mathrm{G}$ is a $\Sigma_{2}^{1}$ graph then either there is a constructible sequence of $\left(\omega_{1}+1\right)$-Borel codes $C_{\gamma}, \gamma<\omega_{1}$, such that the sets $\left[C_{\gamma}\right]$ are $\mathrm{G}$-discrete and cover $\mathcal{N}$, or $\mathrm{G}_{0} \leq_{\mathrm{c}} \mathrm{G}$. However, it is not clear whether the same result can be obtained in the case when the given graph is $\omega_{1}$-L-Suslin in V.

8. Applications for analytic graphs. In this section the technique used above in the proof of Theorem 2 is applied for a different purpose. We prove a form of Theorem 1 which does not seem to be an easy corollary of Theorem 1 itself.

Theorem 19. Let $\lambda<\omega_{1}$. Suppose that $R \subseteq(\omega \times \omega \times \lambda)^{<\omega}$ is a tree such that $\mathrm{G}=\mathfrak{p}[R]$ is a graph on $\mathcal{N}=\omega^{\omega}$. Then $\mathrm{G}$ satisfies one of the following two statements:

(I) $\mathrm{G}$ is countably Borel colourable. Moreover, $\mathcal{N}$ is covered by a union of $\mathrm{L}[R]$-countably many $\mathrm{G}$-discrete $(\lambda+1)-\mathrm{L}[R]$-Borel sets.

(II) $\mathrm{G}_{0} \leq_{\mathrm{c}} \mathrm{G}$. Moreover, $\mathrm{G}_{0} \sqsubseteq_{\mathrm{c}} \mathrm{G}$ provided $\mathrm{G}$ is acyclic.

Note that (I) and (II) are incompatible: this easily follows from Theorem 1.

To see that the theorem is not formally covered by Theorem 1 consider the case when $\lambda \geq \omega_{1}^{\mathrm{L}[R]}$. Then the graph $\mathrm{G}=\mathfrak{p}[R]$ may not be $\Sigma_{1}^{1}\left(z_{0}\right)$ for a real $z_{0} \in \mathrm{L}[R]$.

It is not clear whether one gets $\mathrm{G}_{0} \sqsubseteq_{\mathrm{c}} \mathrm{G}$ in the locally countable case as well. (Note that locally thin $\boldsymbol{\Sigma}_{1}^{1}$ graphs are locally countable.) The method how we treated locally thin graphs above does not seem adequate.

Pro of (of Theorem 19). We follow the general scheme applied above. Let us suppose that $R \in \mathrm{L}$; otherwise L changes to $\mathrm{L}[R]$ in the reasoning.

Let $\mathrm{V}$ denote the universe of Theorem $19 . \mathrm{V}^{+}$will be the $\omega_{2}^{\mathrm{L}[R]}$-collapse extension of $\mathrm{V}$. We finally set $\mathrm{G}^{+}=\mathfrak{p}[R]$ in $\mathrm{V}^{+}$.

Define, in $\mathrm{V}^{+}, \mathcal{C}$ to be the collection of all $(\lambda+1)$-Borel codes $C \in \mathrm{L}$ for the space $\mathcal{N}$ such that $[C]$ is a $\mathrm{G}^{+}$-discrete set in $\mathrm{V}^{+}$. 
If $\bigcup_{C \in \mathcal{C}}[C]=\mathcal{N}$ in $\mathrm{V}^{+}$then, using Lemma 11, we obtain a subcollection $\mathrm{C}^{\prime} \subseteq \mathrm{C}, \mathrm{C}^{\prime} \in \mathrm{L}$, of cardinality $\leq \lambda$ (therefore countable) in $\mathrm{L}$, such that still $\mathcal{N}=\bigcup_{C \in \mathcal{C}^{\prime}}[C]$ in $\mathrm{V}^{+}$, hence in $\mathrm{V}$, which easily leads to (I). Thus we may assume that $\mathfrak{X}=\complement \complement \bigcup_{C \in \mathcal{C}}[C] \subseteq \mathcal{N}$ is non-empty in $\mathrm{V}^{+}$.

In this assumption we define, in $\mathrm{V}$, a system of finite sequences $\tau_{u} \in \omega^{<\omega}$ $\left(u \in 2^{<\omega}\right.$ ) and $\sigma_{u v} \in \lambda^{<\omega}$ (where $\langle u, v\rangle \in \mathrm{CP}_{m}$ for some $m$ ) satisfying the following three requirements for all $m$ :

(i) $\tau_{u} \subset \tau_{u^{\wedge} i}$ for all $u \in 2^{m}$ and $i=0,1$, and $\sigma_{u v} \subset \sigma_{u^{\wedge} i, v^{\wedge} i}$ for all $\langle u, v\rangle \in \mathrm{CP}_{m}$ and $i=0,1$;

(ii) if $\langle u, v\rangle \in \mathrm{CP}_{m}$ then $\left\langle\tau_{u}, \tau_{v}, \sigma_{u v}\right\rangle \in R$;

(iii) there exists, in $\mathrm{V}^{+}$, a system of reals $x_{u} \in \mathcal{N}_{\tau_{u}} \cap \mathfrak{X}\left(u \in 2^{m}\right)$ and functions $f_{u v} \in \lambda^{\omega}\left(\langle u, v\rangle \in \mathrm{CP}_{m}\right)$ such that, for any pair $\langle u, v\rangle \in \mathrm{CP}_{m}$, we have $\sigma_{u v} \subset f_{u v}$ and $\left\langle x_{u}, x_{v}, f_{u v}\right\rangle \in[R]$.

The existence of such a system implies $G_{0} \leq_{c} G$ in $V$, as above. (If $G$ is acyclic in $\mathrm{V}$ then requirement (iv) of Subsection 4.3 joins the list (i)(iii).) The construction itself is pretty much similar to the construction in Section 5 , and is left to the reader.

Acknowledgements. I would like to thank A. S. Kechris and G. Hjorth for useful information relevant to the topic of this paper, A. Kanamori for the interest in the paper, and the referee for important remarks and corrections. I am grateful to M. van Lambalgen, M. Reeken and F. Topsoe for hospitality and support during my visits to the universities of Amsterdam, Wuppertal, and Copenhagen in 1996, where this paper was written.

\section{References}

[1] D. Guaspari, Trees, norms, and scales, in: London Math. Soc. Lecture Note Ser. 87, Cambridge Univ. Press, 1983, 135-161.

[2] L. A. Harrington, A. S. Kechris and A. Louveau, A Glimm-Effros dichotomy for Borel equivalence relations, J. Amer. Math. Soc. 3 (1990), 903-928.

[3] L. A. Harrington and S. Shelah, Counting equivalence classes for co- $\kappa$-Souslin equivalence relations, in: D. van Dalen et al. (eds.), Logic Colloquium '80 (Prague, 1980), North-Holland, 1982, 147-152.

[4] G. Hjorth, Thin equivalence relations and effective decompositions, J. Symbolic Logic 58 (1993), 1153-1164.

[5] -, A remark on $\boldsymbol{\Pi}_{1}^{1}$ equivalence relations, handwritten note.

[6] V. Kanovei, An Ulm-type classification theorem for equivalence relations in Solovay model, J. Symbolic Logic, to appear.

[7] - , On a dichotomy related to colourings of definable graphs in generic models, preprint ML-96-10, University of Amsterdam, 1996.

[8] A. S. Kechris, Classical Descriptive Set Theory, Springer, 1995. 
[9] A. S. Kechris, S. Solecki and S. Todorčević, Borel chromatic numbers, Adv. Math., to appear.

[10] R. M. Solovay, A model of set theory in which every set of reals is Lebesgue measurable, Ann. of Math. 92 (1970), 1-56.

Department of Mathematics

Moscow Transport Engineering Institute (MIIT)

Obraztsova 15

Moscow 101475, Russia

E-mail: kanovei@mech.math.msu.su

Received 22 May 1996;

in revised form 7 February 1997 